\title{
Evaluación de la eficiencia en aeropuertos privatizados
}

\author{
Assessment of efficiency in privatized airports \\ Avaliação da eficiência em aeroportos privatizados
}

Luis Pulido Moreno[a] [D, Oscar Díaz Olariaga ${ }^{[b]}$ [iD

\begin{abstract}
[a] Universidad Santo Tomás, Facultad de Ingeniería Industrial, Bogotá, Colombia
\end{abstract}
[b] Universidad Santo Tomás, Facultad de Ingeniería Civil, Bogotá, Colombia

Como citar: Moreno, L. P., \& Olariaga, O. D. (2019). Evaluación de la eficiencia en aeropuertos privatizados. urbe. Revista Brasileira de Gestão Urbana, v.11, e20180210. https://doi.org/10.1290/2175-3369.011.e20180210

\section{Resumen}

Desde mediados de la década de 1990, y en varias fases temporales, se han privatizado los principales aeropuertos de Colombia. En base a ello, resulta de gran interés conocer, analizar e incluso medir la eficiencia técnica de los aeropuertos en un contexto de reforma estructural, es decir, evaluar la influencia de la privatización de los mismos en la eficiencia y en una relación "antes-después". La metodología utilizada para realizar el estudio es el Análisis Envolvente de Datos (DEA). Se utiliza esta metodología para estimar la eficiencia relativa de 16 aeropuertos (ahora ya privatizados) de Colombia y a nivel comparativo, es decir, en tres escenarios específicos consecutivos: gestión pública, transición, gestión privada. La metodología utiliza "variables técnicas" típicas (utilizadas en investigaciones similares). Los principales resultados de la investigación revelan índices de eficiencia global muy superiores cuando la gestión de los aeropuertos es completamente privada (es decir, realizada $100 \%$ por un operador privado) con respecto a cuando eran gestionados por el sector público.

Palabras clave: Gestión de aeropuertos. Análisis Envolvente de Datos. Eficiencia aeroportuaria. Transporte aéreo. Colombia.

\section{Resumo}

Desde a metade da década dos anos 90, e em momentos diferentes, os principais aeroportos da Colômbia foram privatizados. Daquele, é interessante conhecer, analisar e até medir a eficiência técnica dos aeroportos, no contexto dessa reforma estrutural, ou seja, avaliar a influência que a privatização teve sobre eles e sobre sua eficiência, através de um relacionamento "antes-depois". A metodologia utilizada para realizar o estudo foi a Data Envelopment Analysis (DEA). Esta metodologia foi utilizada para estimar a eficiência relativa de 16 aeroportos (agora privatizados) da Colômbia em um nível comparativo, ou seja, em três cenários específicos consecutivos: na gestão estadual, na transição entre gestões e na gestão privada. A metodologia usa "variáveis técnicas típicas" (usadas em investigações similares). Os principais resultados da pesquisa revelam índices de eficiência globais muito maiores quando a gestão de aeroportos é totalmente

LPM es Master Ingeniero Industrial, e-mail: luis.pulido@usantotomas.edu.co

ODO es Doctor Ingeniero Aeronáutico, Doctor en Economía y Administración, e-mail: oscardiazolariaga@usantotomas.edu.co 
privada (ou seja, efetuada $100 \%$ por um operador privado) em comparação a quando eles foram geridos pelo setor público.

Palavras-chave: Gestão de aeroportos. Análise por Envoltória de Dados. Eficiência de aeroportos. Transporte aéreo. Colômbia.

\section{Abstract}

Since the mid-1990s, and in several temporary phases, the main Colombian airports have been privatized. Based on this, it is of great interest to know, analyze and even measure the technical efficiency of airports in a context of structural reform, that is, to evaluate the influence of the privatization of airports on efficiency and a relationship "before-after". The methodology used to carry out the study is the Data Envelopment Analysis (DEA). This methodology is used to estimate the relative efficiency of 16 airports (now privatized) in Colombia and at a comparative level, that is, in three specific consecutive scenarios: public management, transition, private management. The methodology uses typical "technical variables" (used in similar investigations). The main results of the research reveal much higher global efficiency rates when the management of airports is completely private (that is, carried out $100 \%$ by a private operator) with respect to when they were managed by the public sector.

Keywords: Airport management. Data Envelopment Analysis. Airport efficiency. Air transport. Colombia.

\section{Introducción}

La evaluación de la eficiencia aeroportuaria ha sido un área de investigación en auge en los últimos años. Estas evaluaciones son importantes para todos los actores de la industria, operadores de aeropuertos (públicos y privados), organismos reguladores, gobiernos (locales, regionales y nacionales), y aerolíneas. Por ello, en los últimos 15-20 años se han publicado una gran cantidad de artículos sobre eficiencia aeroportuaria (Lo Storto, 2018; Lai et al., 2012). En lo que refiere a las técnicas específicas adoptadas en las investigaciones el Data Envelopment Analysis (DEA) es uno de los más utilizados, empezado por el trabajo de Gillen \& Lall (1997) que fue uno de los primeros en la materia.

Muchas investigaciones, que intentan medir la eficiencia de los aeropuertos, cubren sus análisis a nivel país (o a veces abarcan regiones continentales); por ejemplo se encuentran estudios para aeropuertos de EE.UU. (Sarkis, 2000; Sarkis \& Talluri, 2004), de Australia (Abbott \& Wu, 2002), de Japón (Yoshida \& Fujimoto, 2004), del Reino Unido (Parker, 1999), de España (Martín \& Román, 2001), de Italia (Malighetti et al., 2007; Barros \& Dieke, 2008, 2007), de Turquía (Ülkü, 2015; Kocak, 2011), etc. Y a nivel de regionales continentales, es decir el análisis simultáneo de un conjunto de aeropuertos de diferentes países (e incluso de diferentes continentes y a nivel mundial), se encuentran los trabajos de Adler \& Berechman (2001), Oum et al. (2003, 2004, 2006), Lin \& Hong (2006), Pels et al. (2001, 2003), Tsui et al. (2014), Ha et al. (2010), Lam et al. (2009), Yang (2010a, 2010b). Cabe mencionar que para la región de Latinoamérica se encuentra muy poca literatura, solo se destacan: un estudio de Perelman \& Serebrisky (2012) donde se analizó la eficiencia aeroportuaria de varios aeropuertos de la región; Barros (2008) examinó la eficiencia técnica de los aeropuertos en Argentina, y analizó la resultados en el contexto de la crisis económica durante el período 2003-2007; y finalmente Wanke (2013), Pacheco \& Fernandes (2003), Almeida et al. (2017) y Perico et al. $(2017,2015)$ midieron la eficiencia de aeropuertos en Brasil. No se encuentran investigaciones (al menos publicadas) sobre la medición de la eficiencia en aeropuertos de Colombia.

Entonces, la presente investigación tomará como caso de estudio, para la medición de la eficiencia (técnica) aeroportuaria, un conjunto de aeropuertos de Colombia, los cuales presentan ciertas características que los hacen susceptibles a dicho análisis. El transporte aéreo en Colombia viene experimentado un crecimiento relevante y sostenido desde hace dos décadas, resultado de la liberalización de la industria (a principios de la década de 1990) y que condujo a la entrada de capital privado al sector aeroportuario. Desde mediados de la década de 1990, y en varias fases temporales, se han privatizado los principales aeropuertos del país (19 a la fecha, los más grandes e importantes de la red), entiendo por tal la entrega en concesión (a operadores privados) sólo de la operación / gestión del aeropuerto (nunca la 
propiedad) (Díaz Olariaga, 2017). En base a ello, resulta de gran interés conocer y medir la eficiencia de los aeropuertos en un contexto antes-después, es decir, evaluar la influencia de la privatización de los mismos en la eficiencia. Y la metodología utilizada para realizar el estudio es el Análisis Envolvente de Datos (DEA), que es una metodología utilizada para estimar la eficiencia relativa de una variable sin la necesidad de conocer a profundidad la relación que se puede presentar entre los inputs y los outputs de un sistema complejo.

\section{Revisión de la bibliografía}

En las últimas dos décadas, y a nivel mundial, la academia ha investigado el impacto de diferentes formas de propiedad sobre la eficiencia de los aeropuertos (Lo Storto, 2018; Chen et al., 2017; Lai et al., 2012). Y desde un punto de vista metodológico el Data Envelopment Analysis (DEA) se ha convertido en la herramienta más popular para los análisis y medición de la eficiencia.

Algunos estudios encuentran evidencia clara de la influencia de la gobernanza en la eficiencia del aeropuerto, otros no pueden descubrir ningún efecto relevante, y otros muestran que el efecto de la gobernanza en la eficiencia del aeropuerto depende de muchos otros factores, por ejemplo relacionados con el mercado y el entorno competitivo, la estructura de gobierno (del operador del aeropuerto), el tipo de concesión, y hasta la capacidad del aeropuerto en generar economías de escala. Los investigadores han usado diferentes muestras, variables, períodos de tiempo y métodos de investigación (Lo Storto, 2018). Ciertas discrepancias entre los resultados obtenidos en las investigaciones se deben a las diferencias en el enfoque de la investigación. Se pueden encontrar resultados contradictorios para muestras de aeropuerto iguales o similares en diferentes marcos de tiempo (Gitto \& Mancuso, 2012; Oum et al., 2003, 2006, 2008). Las heterogeneidades en las muestras y los conjuntos de datos hacen problemáticos los estudios comparativos y pueden sesgar fuertemente el análisis de eficiencia porque los entornos operativos de los aeropuertos son muy diferentes (Adler et al., 2013; Marques et al., 2015).

Varias investigaciones respaldan la idea de que la eficiencia aeroportuaria está influenciada por el tipo de gobernanza. En particular, algunos académicos encontraron que la privatización aumenta la eficiencia del aeropuerto (Adler \& Liebert, 2014; Assaf, 2011, 2010; Gitto \& Mancuso, 2012; Malighetti et al., 2007; Marques \& Barros, 2011; Marques et al., 2015; Oum et al., 2006; Perelman \& Serebrisky, 2012). Adler \& Liebert (2014) encontraron que los operadores de aeropuertos de propiedad mixta y con una mayoría pública no son rentables. Malighetti et al. (2007) estudiaron la eficiencia de los aeropuertos italianos aplicando un modelo DEA, ellos encontraron una influencia positiva de la gobernanza privada en la eficiencia del aeropuerto. Marques and Barros (2011) investigaron el efecto que la regulación y la gobernanza de una muestra de varios aeropuertos europeos, su resultados concluyen que los aeropuertos con gobernanza pública son menos eficientes que los aeropuertos con gobernanza privada. Oum et al. (2006) realizaron una extensa investigación sobre varios aeropuertos de Asia-Pacífico, Europa y América del Norte, y con diferentes tipos o fórmulas de gobernanza, su estudio muestra que los aeropuertos con gobernanza mayoritaria del gobierno (pública) y con gobernanza multinivel son menos eficiente que los aeropuertos con una gobernanza mayoritariamente privada. Un trabajo reciente de Lo Storto (2018) encuentra resultados duales, es decir, en primer lugar su investigación afirma que los resultados sugieren que la heterogeneidad de propiedad puede ejercer una influencia sustancial en la eficiencia del aeropuerto; y en segundo lugar, se detecta que mientras que los aeropuertos de estructura PPP tienen una mayor eficiencia técnica que los aeropuertos operados por operadores públicos, el análisis de meta-frontera relativo a la eficiencia de costos e ingresos no proporciona evidencia de que los aeropuertos PPP funcionan mejor. De hecho, sus resultados muestran que los aeropuertos gestionados públicamente logran comparables o mejor desempeño en términos de eficiencia de ingresos.

Sin embargo, es posible encontrar algunas investigaciones cuyos resultados mostraron que aeropuertos con gobernanza públicas eran más eficientes que con gobernanza privada (Curi et al., 2010; Oum et al., 2008; Gutiérrez \& Lozano, 2016; Martini et al., 2013). Y por otro lado, algunos trabajos (Lin \& Hong, 2006; Gitto \& Mancuso, 2012) determinaron que la eficiencia aeroportuaria no estuvo influenciada por el tipo de propiedad del aeropuerto. 
Finalmente, algunos académicos estudiaron el efecto combinado de gobernanza y otros factores (por ejemplo: regulación, competencia en el mercado, tipo de concesión, etc.) sobre la eficiencia del aeropuerto (Adler \& Liebert, 2014; Albalate et al., 2014; Assaf \& Gillen, 2012; Bel \& Fageda, 2010; Curi et al., 2011; Gillen \& Mantin, 2014). Adler \& Liebert (2014) encontraron que en condiciones relativamente no competitivas los aeropuertos con gobernanza pública son menos eficientes que los aeropuertos con gobernanza totalmente privada, mientras que en un entorno competitivo ambos funcionan igual de eficientes. Assaf \& Gillen (2012) evaluaron el impacto conjunto de la estructura de gobierno y regulación económica sobre la eficiencia aeroportuaria en una amplia muestra de aeropuertos Europa, América del Norte y Australia; los autores descubrieron que el tipo de regulación económica afecta la eficiencia más que el tipo de gobernanza. Curi et al. (2011) utilizaron DEA bootstrapped para estimar el eficiencia técnica de un conjunto de aeropuertos italianos y sus resultados les llevaron a afirmar que la tipología del contrato de concesión podría ser una fuente de variación de la eficiencia. Gillen \& Mantin (2014) corroboran estos hallazgos desarrollando un modelo teórico para explorar la compensación entre el tipo de concesión aeroportuaria y los ingresos aeronáuticos; su modelo muestra que la privatización puede no ser recomendada a menos que el potencial de los ingresos de la concesión sean lo suficientemente grandes.

\section{Caso de estudio}

Sobre la gestión de la infraestructura aeroportuaria Colombia ha seguido la tendencia regional de concesionar la administración de dichas infraestructuras (Díaz Olariaga \& Ávila, 2015; Serebrisky, 2012; Bosch and García Montalvo, 2003), como son los casos de Brasil (Espirito Santo, 2013), Perú (Mendiola et al., 2011), Chile (Carvallo, 2008), Argentina (Lipovich, 2008), México (Rico Galeana, 2008), entre otros. Este modelo busca liberar al Estado del alto gasto requerido por la infraestructura aeronáutica. En Colombia este proceso está regulado por una ley nacional (Ley 80 de 1993, artículo 30 (Congreso de la República, 1993)). Por otra parte, el Gobierno, a través de otra norma (DNP, 1994), consagró el proceso de concesiones de aeropuertos a través de un plan de ordenamiento de la infraestructura aeroportuaria. El documento además de plantear una renovación tecnológica, autorizó el proceso de concesión de los aeropuertos a empresas privadas, y consideró la contraprestación económica para el Estado como único elemento para entregar la concesión. A esta contraprestación se le definió como destino: a) alimentar el fondo de compensación aeronáutica para subsidiar a los aeropuertos no rentables, b) financiar nuevas inversiones en aeropuertos y c) financiar los servicios de tráfico aéreo y seguridad (Díaz Olariaga, 2017, 2016a, 2016b).

Entonces, desde mediados de la década de 1990, y en varias fases temporales, denominadas generaciones, el gobierno colombiano entregó en concesión varios aeropuertos del país, un total de 19 a la fecha, incluidos los más grandes e importantes (y que gestionan el grueso del tráfico aéreo de toda la red) de los 75 que administra la autoridad pública aeronáutica (Díaz Olariaga, 2017, 2016a), con el fin de obtener una mejor administración, modernización y expansión, operación, explotación comercial y mantenimiento de las terminales aéreas de mayor uso. Como resultado de las políticas públicas, tanto de privatización como de inversión pública y privada en infraestructura aeroportuaria, y acompañadas con políticas de desregulación del sector aerocomercial, donde las tarifas aéreas está totalmente liberalizadas desde el año 2012 (Díaz Olariaga \& Zea, 2018), en las últimas dos décadas y media el transporte de pasajeros (totales) creció un $863 \%$ (Díaz Olariaga, 2018).

En otro orden, se puede afirmar que el transporte aéreo en Colombia prácticamente no tiene competencia (a nivel doméstico) con otros medios de transporte, sobre todo para distancias medias y largas, debido a dos situaciones determinantes: primero, la compleja geografía del país (atravesado de suroeste a noreste por tres cadenas montañosas de la Cordillera de Los Andes), y segundo, a la deficiencia (en cobertura, capacidad y tecnología) de los sistemas de comunicación terrestre (no existencia de autopistas de media o alta capacidad) y ferroviario existentes (no existencia de sistema / red de trenes de pasajeros) (Díaz Olariaga \& Carvajal, 2016). Y finalmente, y por la distribución geográfica de los aeropuertos en el país, y por la estructura de las concesiones aeroportuarias (Díaz Olariaga, 2017), no existe competencia intra-aeroportuaria. 
Entonces, en la Tabla 1 se presenta la información de los aeropuertos privatizados y que forman parte de la presente investigación. Mencionar que luego de la entrega del aeropuerto al operador privado, los mismos verificaron una importante modernización tecnológica y de infraestructura, realizados en tiempos muy diferentes según el tamaño del aeropuerto y el volumen de inversión inicial (obligado por contrato de concesión).

Tabla 1 - Datos de aeropuertos con gobernanza 100\% privada

\begin{tabular}{cccc}
\hline Ciudad del aeropuerto & Código IATA & Año de privatización & PAX (2017) \\
\hline Carepa & APO & 2008 & 205362 \\
Medellín & EOH & 2008 & 1070158 \\
Rionegro & MDE & 2008 & 7325740 \\
Barranquilla* & BAQ & 2015 & 2576253 \\
Cartagena & CTG & 2010 & 4590151 \\
Valledupar & VUP & 2010 & 387634 \\
Quibdó & UIB & 2008 & 372618 \\
Montería & MTR & 2008 & 938460 \\
Bogotá & BOG & 2007 & 24694288 \\
Riohacha & RCH & 2010 & 149980 \\
Santa Marta & SMR & 2010 & 1686025 \\
Cúcuta & CUC & 2010 & 875519 \\
Providencia & PVA & 2007 & 71091 \\
San Andrés & ADZ & 2007 & 2328104 \\
Barrancabermeja & EJA & 2010 & 128895 \\
Bucaramanga & BGA & 2010 & 1565482 \\
Corozal & CZU & 2008 & 82675 \\
Cali & CLO & 2000 & 4858057 \\
\hline
\end{tabular}

Nota: "El aeropuerto de la ciudad de Barranquilla (BAQ) no participa del estudio ya que fue privatizado recientemente (y no se cuenta con datos recientes suficientes para el análisis). Fuente: Aerocivil (2018).

Finalmente, en la Figura 1 (a, b, c, d, e, f) se presenta el desarrollo del tráfico de pasajeros de todos los aeropuertos analizados durante el periodo de estudio, el cual comprende las fases temporales de gobernanza pública y privada.


Figura la - Desarrollo del tráfico de pasajeros (totales) en el periodo de estudio; entre paréntesis la fecha de privatización del aeropuerto. Fuente: Aerocivil (2018). 

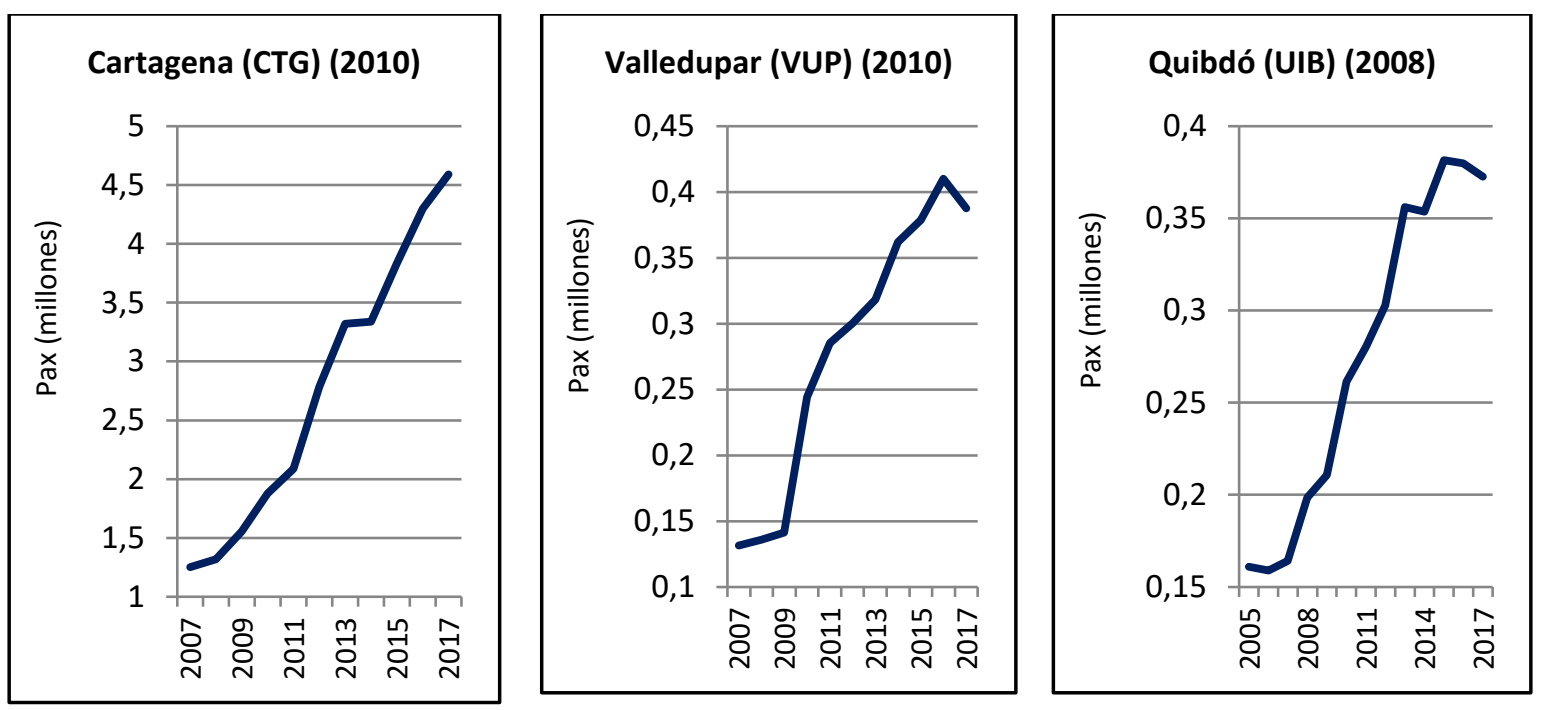

Figura 1b - Desarrollo del tráfico de pasajeros (totales) en el periodo de estudio; entre paréntesis la fecha de privatización del aeropuerto. Fuente: Aerocivil (2018).
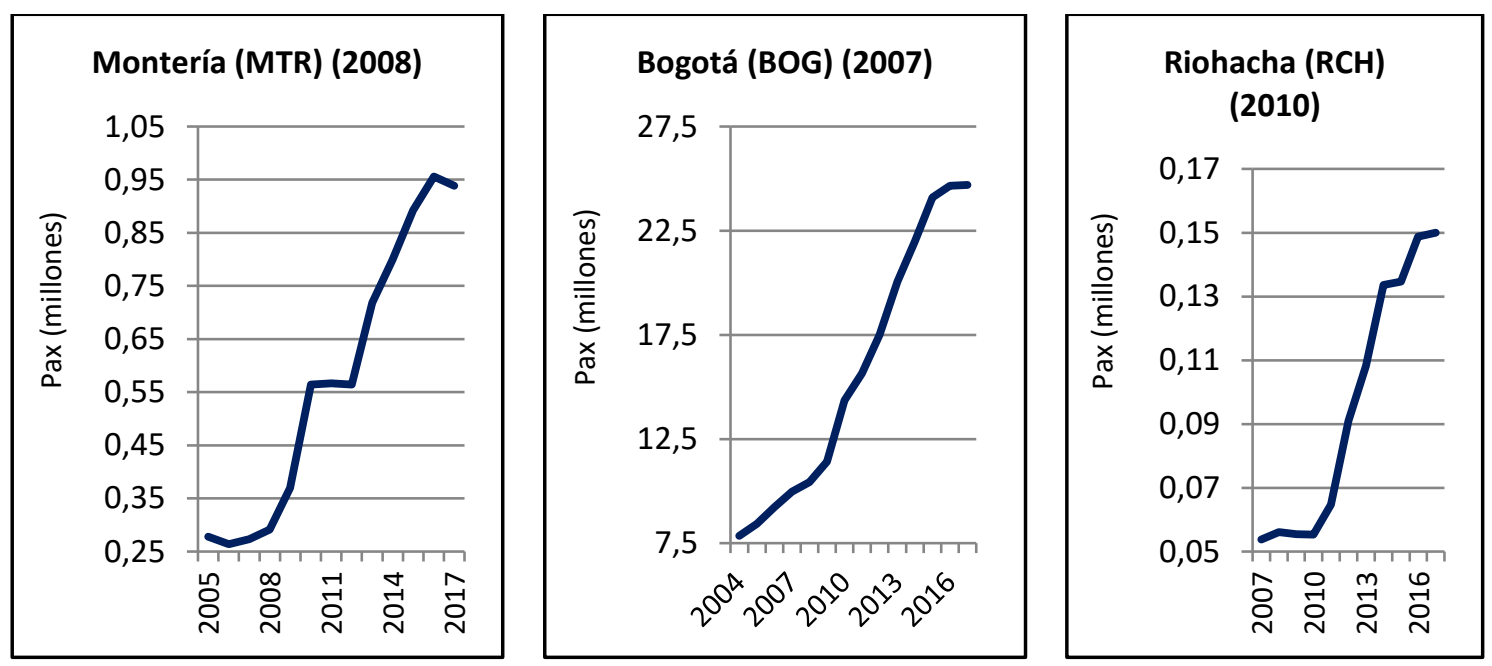

Figura 1c - Desarrollo del tráfico de pasajeros (totales) en el periodo de estudio; entre paréntesis la fecha de privatización del aeropuerto. Fuente: Aerocivil (2018).
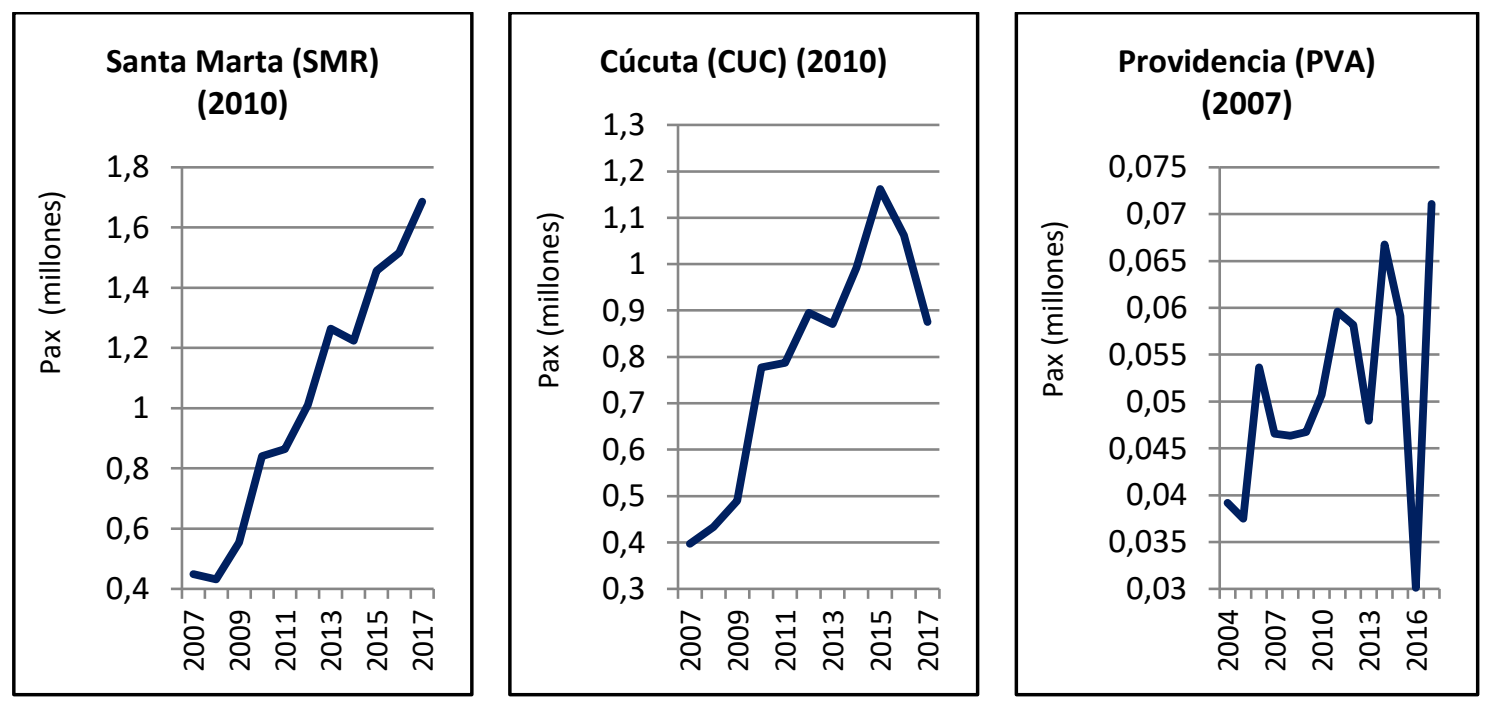

Figura 1d - Desarrollo del tráfico de pasajeros (totales) en el periodo de estudio; entre paréntesis la fecha de privatización del aeropuerto. Fuente: Aerocivil (2018). 

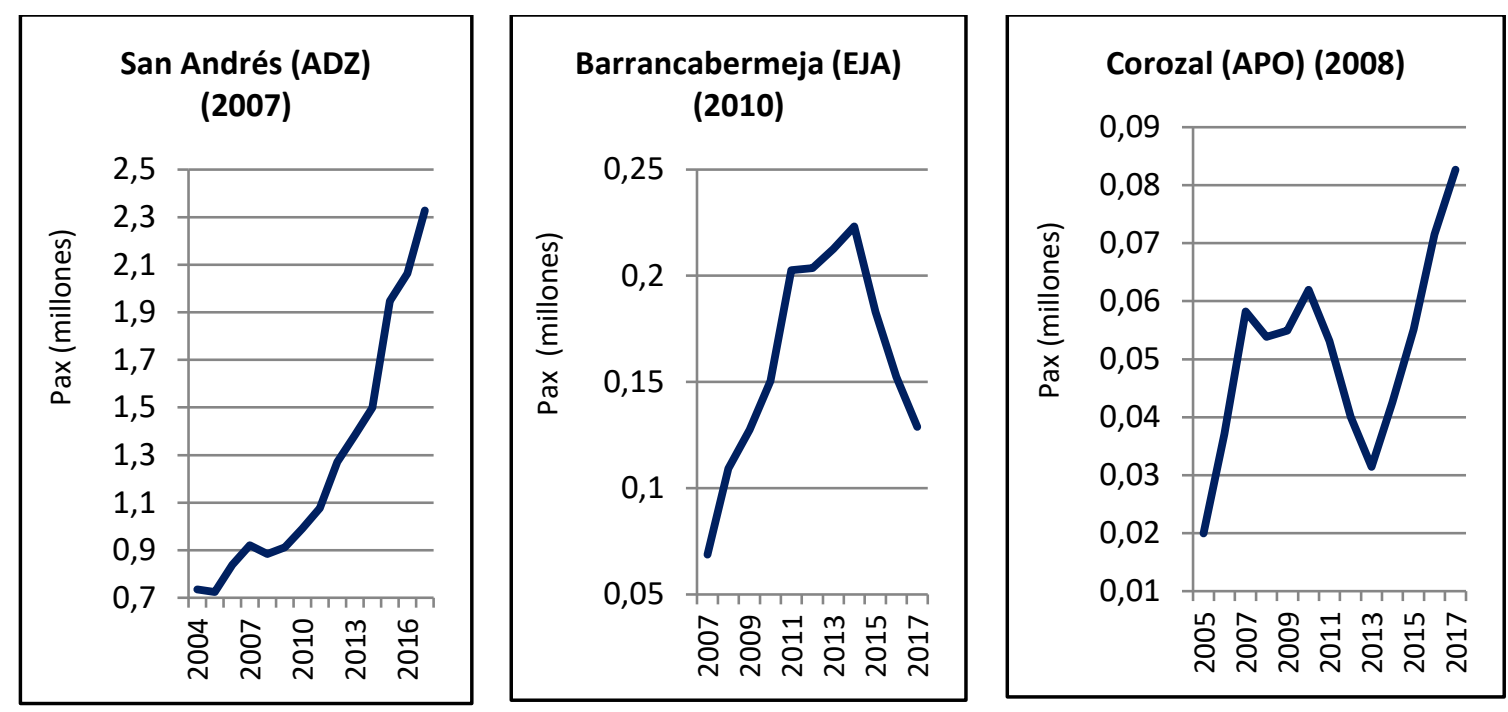

Figura le - Desarrollo del tráfico de pasajeros (totales) en el periodo de estudio; entre paréntesis la fecha de privatización del aeropuerto. Fuente: Aerocivil (2018).


Figura if - Desarrollo del tráfico de pasajeros (totales) en el periodo de estudio; entre paréntesis la fecha de privatización del aeropuerto. Fuente: Aerocivil (2018).

\section{Metodología}

\section{Fundamentos}

La metodología de Análisis Envolvente de Datos (que surge a raíz de la tesis doctoral de Rhodes (1978) y puede considerarse como una extensión del trabajo de Farrell (1957)), que se utiliza en la presente investigación, es considerada como una solución no paramétrica para estimar la productividad de los factores de entrada en un sistema y los resultados de su transformación dentro del proceso con el uso de la programación lineal (Charnes et al., 1978, 1994). El primer modelo DEA, propuesto por Charnes, Cooper y Rhodes (1978), denominado DEA-CCR, tuvo una orientación de entrada y suponía la existencia de rendimientos constantes de escala. El segundo modelo DEA propuesto, conocido como DEA-BCC (Banker et al., 1984), presenta la hipótesis de rendimientos variables de escala. Además de estos dos modelos importantes, hay otros modelos DEA menos frecuentes en la literatura. Así, se identifican al menos otros cinco modelos DEA básicos: el modelo aditivo (Charnes et al., 1985), el modelo multiplicativo (Charnes et 
al., 1982), el modelo DEA cone-ratio (Charnes et al., 1990), el modelo DEA de la región de seguridad (Thompson et al., 1986, 1990) y el modelo de super-eficiencia (Andersen \& Petersen, 1993).

Entonces, en su trabajo Charnes et al. (1978) afirman que frente a un conjunto de unidades consideradas como productivas, y que se pueden comparar entre sí dado que su proceso de transformación de recursos es similar (y para definir un marco de comparación), nombran estas unidades productivas como DMU (Unidades de Toma de Decisión) y definen tres tipos de eficiencia para lograr esta comparación:

(a) Eficiencia técnica: se elige como unidad de referencia la de mayor productividad entre unidades del mismo tamaño.

(b) Eficiencia de escala: refleja la habilidad de la DMU de usar los recursos o factores en proporciones óptimas.

(c) Eficiencia global: se escoge como unidad de referencia la de mayor productividad de las que estén en el estudio.

Las relaciones de eficiencia se miden con el coeficiente obtenido entre los resultados de salida del sistema (outputs) y los recursos que lo abastecieron en el inicio (inputs), con el siguiente criterio (Charnes et al., 1981):

(a) Inputs orientados: buscan, dado el nivel de outputs, la máxima reducción proporcional en el vector de inputs mientras permanece en la frontera de posibilidades de producción. Una unidad no es eficiente si es posible disminuir cualquier input sin alterar sus outputs.

(b) Outputs orientados: buscan, dado el nivel de inputs, el máximo incremento proporcional de los outputs, permaneciendo dentro de la frontera de posibilidades de producción. Una unidad no es eficiente si es posible incrementar cualquier outputs sin incrementar ningún input y sin disminuir ningún otro output.

En general, se sugieren dos tipos de modelos para los estudios de cálculo de la eficiencia:

- Modelo CCR: maximizar un cociente equivale a hacer máximo su numerador si su denominador permanece constante, y cuando un cociente es menor que la unidad es porque el numerador es menor que el denominador.

- Modelo BCC: consiste en la resolución de " $n$ " problemas de maximización correspondientes a cada una de las unidades cuya eficiencia se quiere evaluar. La función objetivo elige los pesos que hacen máxima la eficiencia de la DMU que se estudia.

\section{Conceptualización metodológica}

En la presente investigación se entiende como "eficiencia global" (o "eficiencia técnica", que se usará de forma indistinta) la capacidad de cada aeropuerto analizado de aprovechar productivamente sus recursos de entrada, independiente de su tamaño en comparación con otras unidades dentro del estudio. Para iniciar el cálculo de la eficiencia global de los aeropuertos de estudio, y en concordancia con la literatura existente, se decidió que cada aeropuerto sería medido como una DMU (Unidad Productiva) y para cada uno de los mismos se definieron las variables que se tendrían en cuenta en el análisis como variables de entrada y salida, todas ellas conocidas como "variables técnicas", es decir, las variables de entrada asociadas a la infraestructura existente (y sus características) en el aeropuerto, y las variables salida asociadas a las operaciones aeronáuticas (ver Tabla 2), habitualmente utilizadas en investigaciones similares (ver compendios en: Lo Storto (2018), Gutiérrez \& Lozano (2016), Wanke et al. (2016), Ahn \& Min (2014)). En la presente investigación no se utilizan "variables financieras" u otras de su tipo (de rendimiento, segmentación de ingresos, explotación comercial, productividad, etc.) debido que dicha información, considerada confidencial, los operadores aeroportuarios locales no la suministran. 
Tabla 2 - Definición de variables de entrada y salida del análisis

\begin{tabular}{|c|c|c|}
\hline DMU & Inputs & Outputs \\
\hline $\begin{array}{c}\text { Aeropuertos: } \\
\text { ADZ, SMR, CTG, PVA, MDE, } \\
\text { CLO, APO, MTR, VUP, RCH, } \\
\text { BOG, CZU, UIB, EJA, BGA, } \\
\text { EOH, CUC }\end{array}$ & $\begin{array}{l}\text { 1. Número de pistas. } \\
\text { 2. Longitud de las pistas. } \\
\text { 3. Número de posiciones de estacionamiento de } \\
\text { aeronaves en plataforma. } \\
\text { 4. Área edificada del edificio terminal de pasajeros. } \\
\text { 5. Área de la plataforma. }\end{array}$ & $\begin{array}{l}\text { 1. Operaciones } \\
\text { (despegues/aterrizajes) } \\
\text { 2. Pasajeros transportados. } \\
\text { 3. Carga aérea transportada. }\end{array}$ \\
\hline
\end{tabular}

Fuente: Autor (2018).

Los escenarios definidos para el análisis son:

a) El escenario inicial de comparación de las eficiencias de las terminales aéreas bajo la administración o gestión pública, en este caso se consideró un periodo de tres años, específicamente los últimos tres años en que cada aeropuerto estuvo subordinado a la gestión pública antes de ser privatizado.

b) El segundo escenario denominado de transición: se consideran los dos siguientes años a la entrada en vigencia de la concesión en cada aeropuerto; considerando éste un periodo idóneo para conseguir nivelar la operación a los estándares de cada empresa contratante.

c) El tercer escenario o final: se consideran los últimos cuatro años calendario (a la fecha), es decir 2014, 2015, 2016 y 2017, para cada aeropuerto, lapso en que cada una de ellos ha pasado un periodo considerable bajo la gestión de los operadores privados.

La calificación de los aeropuertos, en nivel de importancia, se realizó a través de un análisis de Pareto utilizando el total de pasajeros transportados en un lapso de once años, considerada ésta como la variable de salida más representativa, considerando para el presente estudio que en Colombia hay cuatro aeropuertos de primer nivel, cinco de nivel intermedio y nueve de nivel bajo; estos niveles son sólo estándares propios en el presente estudio y están relacionados con tráfico de pasajeros anuales gestionados (ver Tabla 1) (no hacen referencia comparativa con aeropuertos de otros países, ni tampoco es un estándar internacional).

Finalmente, y siguiendo la tendencia de la literatura científica existente se decide utilizar el modelo DEA-CCR, el más usado para este tipo de estudios (Charnes et al., 1978), que ofrece la posibilidad de obtener rendimientos a escala comparativa.

\section{Planteamiento del modelo DEA - CCR}

\section{El modelo básico}

Inicialmente el modelo CCR fue propuesto por Charnes et al. (1978). La medida de eficiencia que adoptaron relacionó la suma ponderada de inputs con la de outputs de cada unidad de decisión (DMU) y utilizó modelos de optimización lineal para calcular las ponderaciones. Entonces, el modelo es fraccional y tal cual se presenta en la Ecuación 1.

$$
\operatorname{Max}_{u, v} h o=\frac{\sum_{r=1}^{S} U_{r} * Y_{r o}}{\sum_{i=1}^{m} V_{i} * X_{i o}}
$$

sujeto a:

$$
\frac{\sum_{r=1}^{s} U_{r} * Y_{r j}}{\sum_{i=1}^{m} V_{i} * X_{i j}} \leq 1 \forall j: 1 . . n
$$


$U_{r}, V_{i} \geq 0 \forall r: 1 . .2 \quad \forall i: 1 \ldots . m$

donde:

$h_{o}$ : función objetivo. Medida de la eficiencia.

$Y_{r j}$ : output i-ésimo de la DMU j-ésima.

$X_{i j:}$ input i-ésimo de la DMU j-ésima.

$V_{i}, U_{r}$ : ponderaciones de inputs y outputs respectivamente (soluciones del programa).

\section{Modificaciones del modelo básico}

Esta formulación original fue modificada más tarde (Charnes et al., 1979) con el fin de que las ponderaciones alcanzaran valores estrictamente positivos y, así, evitar que la solución del programa no considerara a todos los factores y productos en el cálculo del índice de eficiencia al tiempo que se evitaba que el denominador del cociente de eficiencia fuera nulo y su valor no existe.

$\operatorname{Max}_{u, v} h o=\frac{\sum_{r=1}^{s} U_{r} * Y_{r o}}{\sum_{i=1}^{m} V_{i} * X_{i o}}$

sujeto a:

$\frac{\sum_{r=1}^{S} U_{r^{*}} Y_{r j}}{\sum_{i=1}^{m} V_{i^{*}} X_{i j}} \leq 1 \forall j: 1 . . n$

$U_{r}, V_{i} \geq \in>0 \quad \forall r, i$

Entonces, el modelo se plantea de la siguiente manera (Tsui et al., 2014):

$B o=\frac{\sum_{r=1}^{m} u_{r} y_{r o}}{\sum_{i=1}^{n} v_{i} x_{i o}}$

sujeto a:

$\frac{\sum_{r=1}^{m} u_{r} y_{r k}}{\sum_{i=1}^{n} v_{i} x_{i o}} \leq 1 \quad \mathrm{~K}=1,2,3 \ldots, \mathrm{m}$

$u_{r}, v_{i \geq 0} \quad \mathrm{r}=1,2,3, \ldots, \mathrm{m} \quad \mathrm{i}=1,2,3, \ldots, \mathrm{n}$

donde:

$B_{0}$ : eficiencia del aeropuerto

$x_{i o}(\mathrm{i}=1,2,3, \ldots, \mathrm{n})$, inputs que producen yro $(\mathrm{r}=1,2,3, \ldots \mathrm{m})$ outputs

$u_{r}$ y $v_{i}$ : multiplicadores virtuales que impiden que el valor de la eficiencia sea menor que cero.

El valor máximo de eficiencia global es 1.0. Un aeropuerto es eficiente totalmente si el cálculo realizado produce exactamente este valor. 


\section{Resultados}

En la Tabla 3 se presentan los resultados obtenidos de la eficiencia global en los últimos tres años de gestión pública de los aeropuertos analizados. En dicho periodo ninguno de ellos se puede considerar como eficiente y el promedio global de este escenario de comparación es de un 52\%.

Tabla 3 - Eficiencia global de los aeropuertos en periodo de gestión pública

\begin{tabular}{ccccc}
\hline DMU & Año 1 & Año 2 & Año 3 & Promedio \\
\hline CLO & $90,90 \%$ & $96,72 \%$ & $89,18 \%$ & $92,27 \%$ \\
EOH & $86,72 \%$ & $87,14 \%$ & $92,15 \%$ & $88,67 \%$ \\
MDE & $79,67 \%$ & $87,04 \%$ & $73,59 \%$ & $80,10 \%$ \\
BOG & $74,56 \%$ & $78,75 \%$ & $80,54 \%$ & $77,95 \%$ \\
UIB & $70,93 \%$ & $66,47 \%$ & $65,10 \%$ & $67,50 \%$ \\
BGA & $56,54 \%$ & $60,19 \%$ & $71,74 \%$ & $62,82 \%$ \\
PVA & $53,39 \%$ & $48,18 \%$ & $62,05 \%$ & $54,54 \%$ \\
EJA & $35,15 \%$ & $48,61 \%$ & $67,05 \%$ & $50,27 \%$ \\
CTG & $48,22 \%$ & $47,47 \%$ & $51,00 \%$ & $48,90 \%$ \\
SMR & $46,58 \%$ & $44,72 \%$ & $47,40 \%$ & $46,23 \%$ \\
ADZ & $41,08 \%$ & $38,80 \%$ & $44,47 \%$ & $41,45 \%$ \\
CZU & $35,90 \%$ & $38,07 \%$ & $36,80 \%$ & $36,92 \%$ \\
APO & $40,03 \%$ & $35,20 \%$ & $35,38 \%$ & $36,87 \%$ \\
CUC & $34,69 \%$ & $33,49 \%$ & $38,95 \%$ & $35,71 \%$ \\
MTR & $27,29 \%$ & $31,95 \%$ & $37,87 \%$ & $32,37 \%$ \\
VUP & $24,90 \%$ & $23,09 \%$ & $25,96 \%$ & $24,65 \%$ \\
RCH & $12,15 \%$ & $10,02 \%$ & $10,27 \%$ & $10,82 \%$ \\
\hline & & Fuente: Autor $(2018)$. &
\end{tabular}

La Figura 2 muestra el comportamiento de los resultados obtenidos. Por debajo de la media global hay diez aeropuertos, y el mejor nivel de eficiencia corresponde al Aeropuerto de Cali (CLO) con un 92\%.



Figura 2 - Eficiencia global de los aeropuertos bajo gestión pública. Fuente: Autor (2018). 
La Tabla 4 muestra los resultados de la eficiencia global obtenida para el escenario de transición. La Figura 3 muestra el comportamiento comparativo de los resultados obtenidos.

Tabla 4 - Eficiencia global de los aeropuertos en el periodo de transición

\begin{tabular}{cccc}
\hline DMU & Año 1 & Año 2 & Promedio \\
\hline EOH & $92,28 \%$ & $87,34 \%$ & $89,81 \%$ \\
BGA & $88,24 \%$ & $84,87 \%$ & $86,55 \%$ \\
UIB & $77,30 \%$ & $82,73 \%$ & $80,01 \%$ \\
BOG & $78,42 \%$ & $76,61 \%$ & $77,51 \%$ \\
PVA & $77,39 \%$ & $75,70 \%$ & $76,55 \%$ \\
EJA & $75,79 \%$ & $68,04 \%$ & $71,92 \%$ \\
CTG & $57,39 \%$ & $73,01 \%$ & $65,20 \%$ \\
CLO & $59,65 \%$ & $62,23 \%$ & $60,94 \%$ \\
SMR & $58,04 \%$ & $59,32 \%$ & $58,68 \%$ \\
MDE & $53,64 \%$ & $62,19 \%$ & $57,92 \%$ \\
ADZ & $51,27 \%$ & $53,66 \%$ & $52,47 \%$ \\
CUC & $44,64 \%$ & $53,91 \%$ & $49,28 \%$ \\
APO & $48,43 \%$ & $44,74 \%$ & $46,59 \%$ \\
MTR & $40,08 \%$ & $42,34 \%$ & $41,21 \%$ \\
VUP & $37,26 \%$ & $35,75 \%$ & $36,51 \%$ \\
CZU & $33,33 \%$ & $34,27 \%$ & $33,80 \%$ \\
RCH & $11,88 \%$ & $15,05 \%$ & $13,46 \%$ \\
\hline
\end{tabular}

Fuente: Autor (2018).

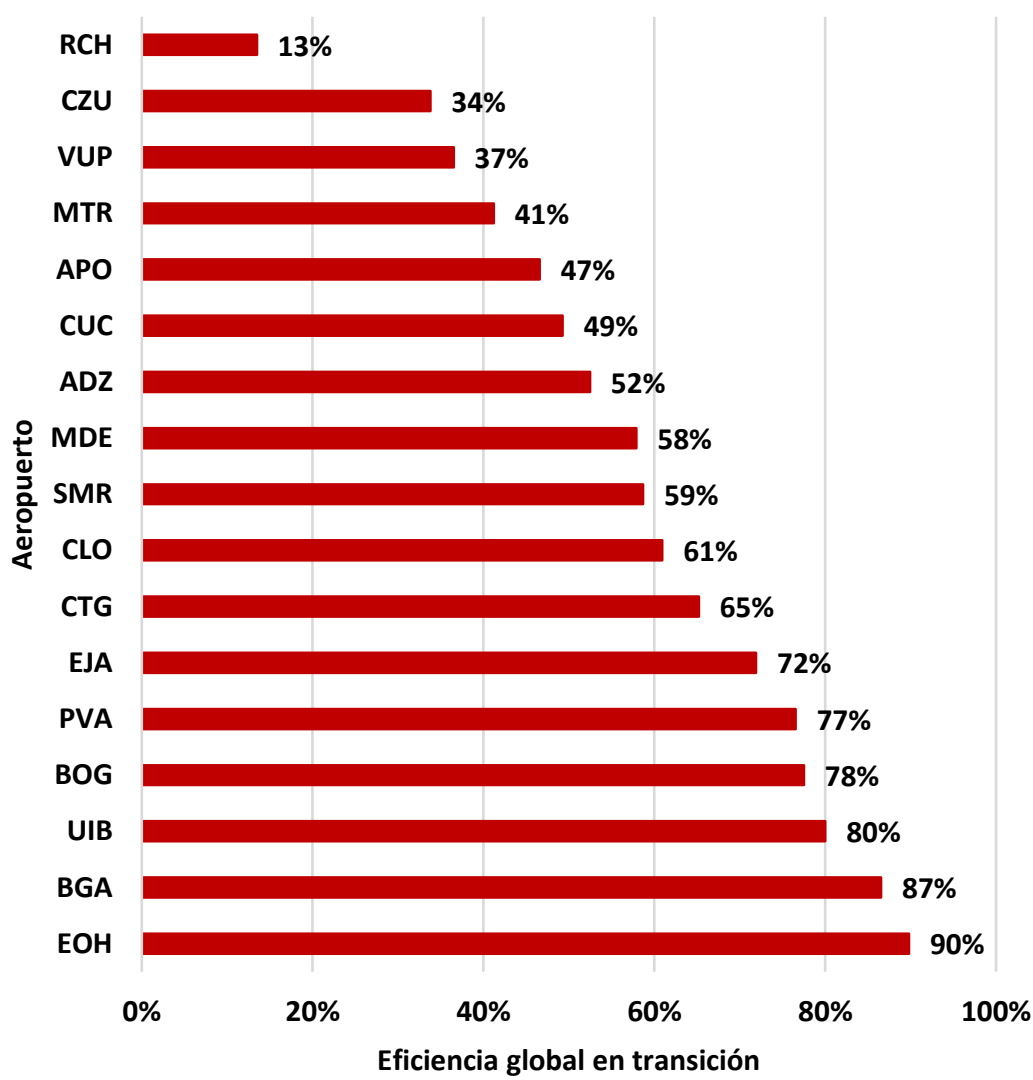

Figura 3 - Eficiencia en el periodo de transición. Fuente: Autor (2018).

En la Tabla 5 se presentan los resultados obtenidos para la eficiencia global de los aeropuertos bajo la gestión privada de los aeropuertos en el periodo 2014-2017. 
Tabla 5 - Eficiencia global de los aeropuertos en el periodo de su gestión privada

\begin{tabular}{cccccc}
\hline DMU & 2014 & 2015 & 2016 & 2017 & Promedio \\
\hline BOG & $92,22 \%$ & $100,00 \%$ & $100,00 \%$ & $100,00 \%$ & $98,06 \%$ \\
MDE & $85,22 \%$ & $100,00 \%$ & $99,32 \%$ & $100,00 \%$ & $96,14 \%$ \\
UIB & $85,40 \%$ & $100,00 \%$ & $98,53 \%$ & $95,50 \%$ & $94,86 \%$ \\
EOH & $95,49 \%$ & $100,00 \%$ & $92,46 \%$ & $88,61 \%$ & $94,14 \%$ \\
CLO & $66,03 \%$ & $100,00 \%$ & $100,00 \%$ & $97,55 \%$ & $90,89 \%$ \\
CTG & $80,02 \%$ & $89,32 \%$ & $94,06 \%$ & $100,00 \%$ & $90,85 \%$ \\
BGA & $96,41 \%$ & $97,15 \%$ & $87,92 \%$ & $81,12 \%$ & $90,65 \%$ \\
APO & $70,93 \%$ & $100,00 \%$ & $88,54 \%$ & $86,54 \%$ & $86,50 \%$ \\
ADZ & $57,82 \%$ & $89,45 \%$ & $93,74 \%$ & $100,00 \%$ & $85,25 \%$ \\
PVA & $66,39 \%$ & $87,54 \%$ & $60,92 \%$ & $96,40 \%$ & $77,81 \%$ \\
SMR & $66,09 \%$ & $75,47 \%$ & $78,53 \%$ & $87,33 \%$ & $76,85 \%$ \\
EJA & $77,70 \%$ & $81,13 \%$ & $68,38 \%$ & $68,15 \%$ & $73,84 \%$ \\
MTR & $46,01 \%$ & $56,00 \%$ & $58,54 \%$ & $58,82 \%$ & $54,84 \%$ \\
CUC & $52,37 \%$ & $58,41 \%$ & $49,22 \%$ & $43,39 \%$ & $50,85 \%$ \\
VUP & $38,31 \%$ & $46,34 \%$ & $49,10 \%$ & $47,23 \%$ & $45,24 \%$ \\
CZU & $35,63 \%$ & $30,30 \%$ & $33,94 \%$ & $38,30 \%$ & $34,54 \%$ \\
RCH & $20,26 \%$ & $23,58 \%$ & $26,00 \%$ & $26,70 \%$ & $24,13 \%$ \\
\hline
\end{tabular}

Fuente: Autor (2018).

En este escenario final (gestión 100\% privada) puede descatarse que:

- En el año 2015 los aeropuertos BOG, MDE, UIB, EOH, CLO y APO alcanzan el nivel máximo de eficiencia global (100\%).

- En el año 2016 solamente los aeropuertos BOG y CLO se mantienen en ese nivel máximo de eficiencia global.

- En el año 2017 los aeropuertos CTG y ADZ consiguen el máximo nivel de eficiencia global, y los aeropuertos de BOG y CLO se mantienen en ese nivel.

- Los aeropuertos BOG y CLO han mantenido el nivel máximo de eficiencia global durante tres años consecutivos, en el periodo 2015-2017.

La Figura 4 muestra los resultados obtenidos para el periodo de gestión 100\% privada de los aeropuertos. El promedio general de la eficiencia global se estima en un 74\%. De los diecisiete aeropuertos en estudio once se ubican por encima de la media global del conjunto.

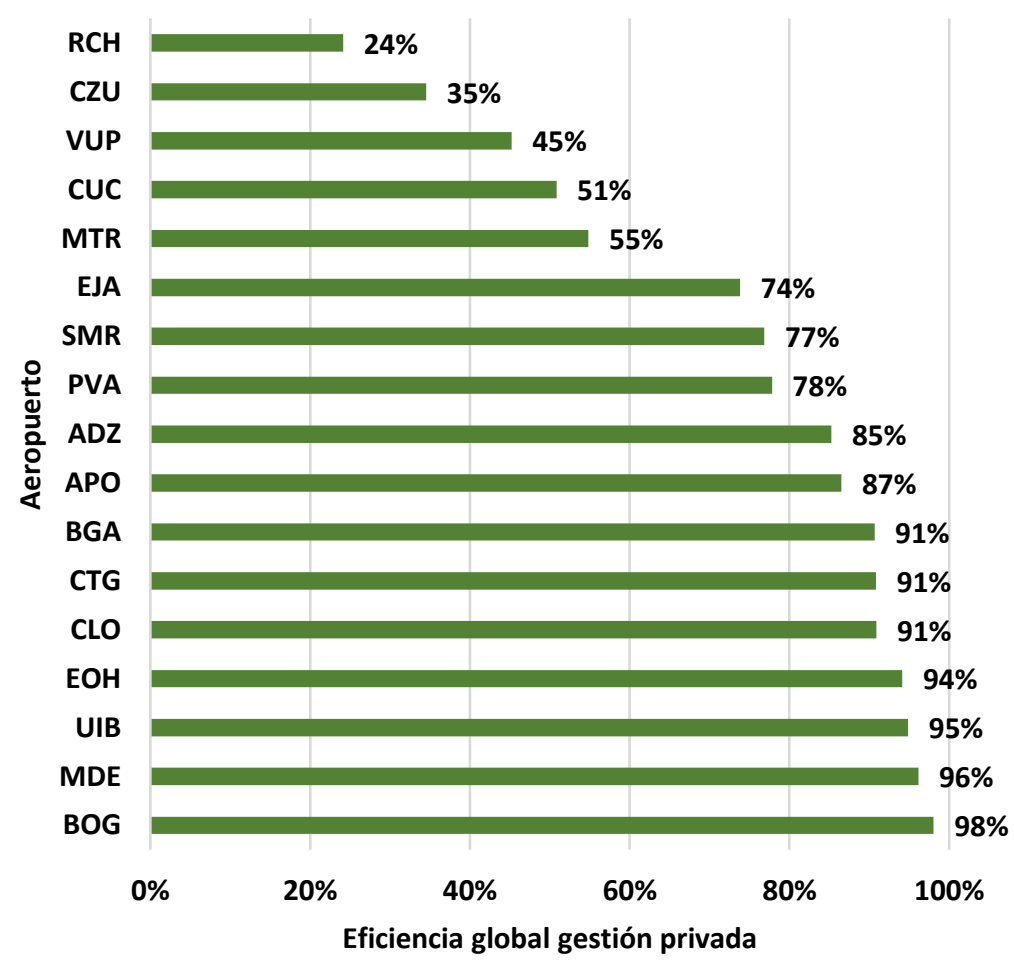

Figura 4 - Eficiencia global de los aeropuertos bajo gestión / operación privada. Fuente: Autor (2018). 
La Figura 5 muestra el cambio de la eficiencia global promedio de los aeropuertos en los tres escenarios planteados.

\section{CAMBIO DE LA EFICIENCIA GLOBAL \\ (Valores promedios)}

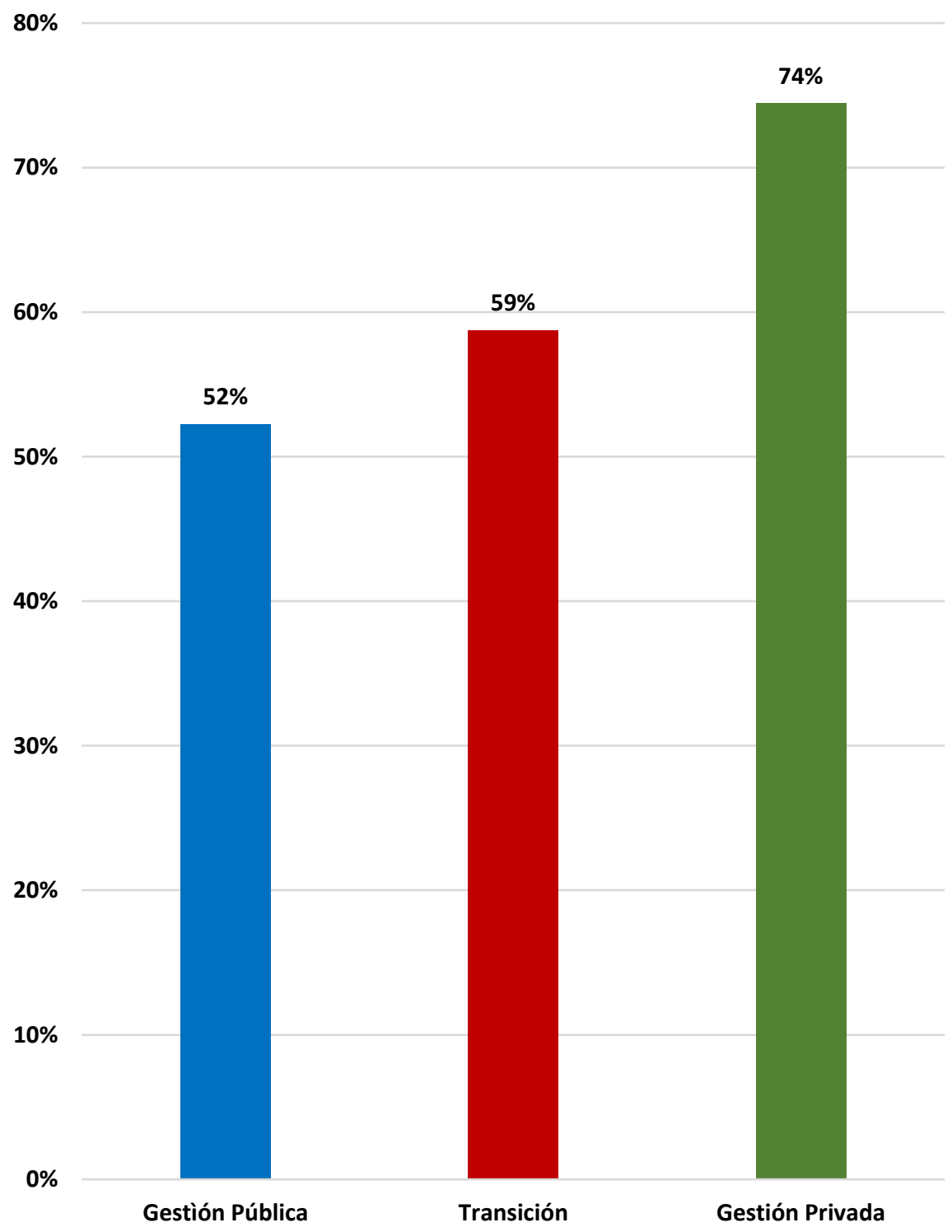

Figura 5 - Comparativa de la eficiencia global del conjunto de aeropuertos analizados en los tres escenarios planteados. Fuente: Autor (2018).

A nivel general se puede apreciar una mejora de un $6 \%$ entre el periodo de transición de gestìon pùblica y por otro lado de un $22 \%$ total en la eficiencia global entre la gestión privada y la publica de los aeropuertos analizados.

La Tabla 6 presenta el cambio porcentual para cada aeropuerto entre la gestión privada y la pública en su eficiencia global. 
Tabla 6 - Cambio de la eficiencia de la gestión privada con respecto a la pública

\begin{tabular}{|c|c|}
\hline DMU & $\begin{array}{c}\text { CAMBIO } \\
\text { PORCENTUAL }\end{array}$ \\
\hline APO & $134,6 \%$ \\
\hline RCH & $123,1 \%$ \\
\hline ADZ & $105,7 \%$ \\
\hline CTG & $85,8 \%$ \\
\hline VUP & $83,6 \%$ \\
\hline MTR & $69,4 \%$ \\
\hline SMR & $66,2 \%$ \\
\hline EJA & $46,9 \%$ \\
\hline BGA & $44,3 \%$ \\
\hline PVA & $42,7 \%$ \\
\hline CUC & $42,4 \%$ \\
\hline UIB & $40,5 \%$ \\
\hline BOG & $25,8 \%$ \\
\hline MDE & $20,0 \%$ \\
\hline EOH & $6,2 \%$ \\
\hline CLO & $-1,5 \%$ \\
\hline CZU & $-6,5 \%$ \\
\hline \multicolumn{2}{|c|}{ Fuente: Autor $(2018)$} \\
\hline
\end{tabular}

\section{Conclusiones}

El presente artículo mide la eficiencia aeroportuaria en un contexto de mercado liberalizado del transporte aéreo, de considerable nivel de inversión global en infraestructura aeroportuaria, pública y privada, de alto crecimiento mantenido en las dos última décadas, y que se prevé mantenga su ritmo actual de desarrollo, al menos a corto plazo (Díaz Olariaga, 2018). Entonces, en esta investigación se ha medido la eficiencia de un conjunto de aeropuertos de la red colombiana, todos ellos con gobernanza $100 \%$ privada, pero con un triple periodo de análisis: gobernanza 100\% pública - transición (luego de la entrega del aeropuerto en concesión privada) - gobernanza 100\% privada. Y a destacar que la metodología adoptada (DEA) ha utilizado para el cálculo solo las denominadas "variables técnicas", es decir, aquellas asociadas / relacionadas a las características de infraestructura (y capacidad) de los aeropuertos y a la actividad aeronáutica (tráfico aéreo). Los resultados obtenidos conducen a las siguientes conclusiones:

a) Los aeropuertos gestionados por el sector privado (por operadores 100\% privados) presentan mejores índices de eficiencia que cuando los mismos eran administrados y gestionados (completamente) por el sector público. Estos resultados van en la misma de línea de muchas investigaciones similares en otros países y regiones del mundo (Lo Storto, 2018).

b) Los aeropuertos, ya privatizados, presentan altos niveles de eficiencia pero solo los grandes aeropuertos (de mayor tráfico aéreo), a excepción del aeropuerto (pequeño) de Quibdó-UIB. Los aeropuertos privatizados pequeños (es de decir de tráfico aéreo muy reducido) presentan niveles de eficiencia muy bajos (excepto UIB antes mencionado).

c) Para el año 2017 (último año del periodo de análisis) solo 4 aeropuertos privatizados presenta un nivel de eficiencia máximo (100\%). En años anteriores algunos aeropuertos alcanzaron el máximo nivel de eficiencia (100\%) pero no lograron mantenerlo.

En definitiva, el presente estudio (el primero en su tipo en Colombia) es una gran primera aproximación al análisis y evaluación de la eficiencia aeroportuaria local. Asimismo es importante avanzar en la cobertura y alcance de la investigación incorporando nuevas variables, además de las técnicas, como son las financieras, de explotación, de rendimiento, variables de origen regulatorio, variables del mercado de la competencia, variables contractuales (relacionadas a los contratos de concesión), etc. Con ello se podría 
conocer y evaluar mucho mejor el comportamiento de la eficiencia, determinar con más exactitud si en realidad en Colombia la privatización mejora la eficiencia aeroportuaria. y hasta se podría generar estándares para ser usados por todos los aeropuertos de la red y poder así realizar un análisis comparativo riguroso entre todos los aeropuertos del sistema.

Sería de gran interés que la autoridad pública regulatoria de Colombia desarrollase, por un lado, estándares para poder medir, evaluar y comparar con precisión los niveles de eficiencia de los aeropuertos de la red, cualquiera sea su gobernanza. Y por otro lado, diseñar e implementar un conjunto (formal) de procedimientos de inspección para realizar los seguimientos y evaluaciones de todos los aeropuertos. Ello facilitaría una sinergia tal que permitiría a los aeropuertos menos eficientes mejorar su indicadores pero sabiendo exactamente cuáles, dónde y cómo realizar procesos de mejora.

\section{Referencias}

Abbott, M. \& Wu S. (2002). Total factor productivity and efficiency of Australian airports. The Australian Economic Review, 35, 244-260.

Adler, N. \& Liebert, V. (2014). Joint impact of competition, ownership form and economic regulation on airport performance and pricing. Transportation Research Part A, 64, 92-109.

Adler, N.; Liebert, V. \& Yazhemsky, E. (2013). Benchmarking airports from a managerial perspective. Omega, 41(2), 442-458.

Adler, N. \& Berechman, J. (2001). Measuring airport quality from the airlines' viewpoint: an application of data envelopment analysis. Transport Policy, 8, 171-181.

Aerocivil (2018). Estadísticas de Transporte Aéreo. www.aerocivil.gov.co

Ahn, Y-H. \& Min, H. (2014). Evaluating the multi-period operating efficiency of international airports using data envelopment analysis and the Malmquist productivity index. Journal of Air Transport Management, 39, 12-22.

Albalate, D.; Bel, G. \& Fageda, X. (2014). Beyond pure public and pure private management models: partial privatization in the European airport industry. International Public Management Journal, 17(3), 308-327.

Almeida, M. R.; Mariano, E. B. \& Rebelatto, D. A. N. (2017). Análise de Eficiência dos Aeroportos Internacionais Brasileiros. Revista Produção, 1-17.

Andersen, P. \& Petersen, N. (1993). A procedure for ranking e_cient units in data envelopment analysis. Management Science, 39(10), 1261-1264.

Assaf, A. \& Gillen, D. (2012). Measuring the joint impact of governance form and economic regulation on airport efficiency. European Journal of Operational Research, 220(1), 187-198.

Assaf, A. (2011). Bootstrapped malmquist indices of Australian airports. The Service Industries Journal, 31(5), 829846.

Assaf, A. (2010). The cost efficiency of Australian airports post privatization: a Bayesian methodology. Tourism Management, 31(2), 267-273.

Banker, R.; Charnes, A. \& Cooper, W. (1984). Some Models for Estimating Technical and Scale Inefficiencies in Data Envelopment Analysis. Management Science, 30(9), 1078-1092.

Barros, C. (2008). Airports in Argentina: technical efficiency in the context of an economic crisis. Journal of Air Transport Management, 14(6), 315-319.

Barros, C. \& Dieke, P. (2008). Measuring the economic efficiency of airports: a Simar-Wilson methodology analysis. Transportation Research Part E, 44, 1039-1051.

Barros, C. \& Dieke, P. (2007). Performance evaluation of Italian airports: a data envelopment analysis. Journal of Air Transport Management, 13, 184-191.

Bel, G. \& Fageda, X. (2010). Privatization, regulation and airport pricing: an empirical analysis for Europe. Journal of Regulatory Economics, 37(2), 142-161. 
Bosch, A. \& García Montalvo, J. (2003). Free and Nondiscriminatory Access to Airports: A Proposal for Latin America. Working Paper, Inter-American Development Bank, Washington, D.C.

Carvallo, C (2008). Experiencia chilena en concesiones aeroportuarias. Santiago de Chile: Ministerio de Obras Públicas.

Charnes, A.; Cooper, W.; Lewin, A. \& Seiford, L. (1994). Data Enveloment Analysis: Theory, Methodology and Applications. New York: Kluwer Academic Publishers.

Charnes, A.; Cooper, W.; Huang, M. \& Sun, D. (1990). Polyhedral Cone-Ratio DEA Models with an Illustrative Application to Large Commercial Banks. Econometrics, 46, 73-91.

Charnes, A.; Cooper, W.; Golany, B.; Seiford, L. \& Stutz, J. (1985). Foundations of data envelopment analysis for ParetoKoopmans efficient empirical production functions. Journal of Econometrics, 30, 91-107.

Charnes, A.; Cooper, W.; Seiford, L. \& Stutz, J. (1982). Multiplicative Model for Efficiency Analysis. Socio-Economic Planning Sciences, 16, 223-235.

Charnes, A.; Cooper, W. \& Rhodes, E. (1981). Evaluating program and managerial efficiency: An application of data envelopment analysis to program follow through. Management Science, 27(6) 668-697.

Charnes, A.; Cooper, W. \& Rhodes, E. (1979). Short communication: measuring the efficiency of decision making units. European Journal of Operational Research, 3(4), 339.

Charnes, A.; Cooper, W. \& Rhodes, E. (1978). Measuring the efficiency of decision making units. European Journal of Operational Research, 2(6), 429-444.

Chen, Y.; Lai, P. \& Piboonrungroj, P. (2017). The relationship between airport performance and privatisation policy: A nonparametric metafrontier approach. Journal of Transport Geography, 62, 229-235.

Congreso de la República (1993). Ley 80 de 1993. Estatuto General de Contratación de la Administración Pública. Bogotá (Colombia).

Curi, C.; Gitto, S. \& Mancuso, P. (2011). New evidence on the efficiency of Italian airports: A bootstrapped DEA analysis. Socio-Economic Planning Sciences, 45, 84-93.

Curi, C.; Gitto, S. \& Mancuso, P. (2010). The Italian airport industry in transitino: a performance analysis. Journal of Air Transport Management, 16, 2018-2221.

Díaz Olariaga, O. (2018). Prognosis de tráfico aéreo en contexto de post-liberalización del transporte aéreo. Caso de Colombia. Working Paper. doi: 10.13140/RG.2.2.11443.73760

Díaz Olariaga, O. (2017). Políticas de privatización de aeropuertos. El caso de Colombia. Documentos y Aportes en Administración Pública y Gestión Estatal, 29, 7-35.

Díaz Olariaga, 0. (2016a). Análisis del desarrollo reciente del transporte aéreo en Colombia. Revista Transporte y Territorio, 14, 122-143.

Díaz Olariaga, O. (2016b). Análisis de la evolución de las políticas públicas y de regulación en la industria aeroportuaria en Colombia. Documentos y Aportes en Administración Pública y Gestión Estatal, 26, 7-42.

Díaz Olariaga, O. \& Zea, J.F. (2018). Influence of the liberalization of the air transport industry on configuration of the traffic in the airport network. Transportation Research Procedia, 33, 43-50.

Díaz Olariaga, O. \& Carvajal, A.F. (2016). Efectos de la liberalización en la geografía del transporte aéreo en Colombia. Cuadernos Geográficos, 55(2), 344-364.

Díaz Olariaga, O. \& Ávila, J. (2015). Evolution of the airport and air transport industry in Colombia and its impact on the economy. Journal of Airline and Airport Management, 5(1), 39-66.

DNP (1994). Reordenamiento institucional y plan de expansión del sistema aeroportuario - Documento CONPES 2727. Bogotá: Departamento Nacional de Planeación.

Espirito Santo, R. A. (2013). Airport privatization and Business Models: What Brazil has and what Brazil Needs. Montreal: VI WALA Conference.

Farrell, M. (1957). The measurement of productive efficiency. Journal of the Royal Statistical Society, Series A, 3, 253290. 
Gillen, D. \& Mantin, B. (2014). The importance of concession revenues in the privatization of airports. Transportation Research Part E, 68, 164-177.

Gillen, D. \& Lall, A. (1997). Developing measures of airport productivity and performance: an application of data envelopment analysis. Transportation Research Part E, 33(4), 261-273.

Gitto, S. \& Mancuso, P. (2012). Two faces of airport business: a non parametric analysis of the Italian airport industry. Journal of Air Transport Management, 20, 39-42.

Gutiérrez, E. \& Lozano, S. (2016). Efficiency assessment and output maximization possibilities of European small and medium sized airports. Research in Transportation Economics, 56, 3-14.

Ha, H.K.; Yoshida, Y. \& Zhang, A. (2010). Comparative analysis of efficiency for major Northeast Asia airports. Transportation Journal, 49(4), 9-23.

Kocak, H. (2011). Efficiency examination of Turkish airports with DEA approach. International Business Research, 4(2), 204-212.

Lai, P.; Potter, A. \& Beynon, M. (2012).The development of benchmarking techniques in airport performance evaluation research. Transportation Journal, 51(3), 305-337.

Lam, S.W.; Low, J.M.W. \& Tang, L.T. (2009). Operational efficiency across Asia-Pacific airports. Transportation Research Part E, 45(4), 654-665.

Lin, L. \& Hong C. (2006). Operational performance evaluation of international major airports: an application of data envelopment analysis. Journal of Air Transport Management, 12, 342-351.

Lipovich, G. (2008). The privatization of Argentine airports. Journal of Air Transport Management, 14(1), 8-15.

Lo Storto, C. (2018). Ownership structure and the technical, cost, and revenue efficiency of Italian airports. Utilities Policy, 50, 175-193.

Malighetti, P.; Martini, G.; Paleari, S. \& Redondi, R. (2007). An Empirical Investigation on the Efficiency, Capacity and Ownership of Italian Airports. Rivista di Politica Economica, 47(I-II), 157-188.

Marques, R.C.; Simoes, P. \& Carvalho, P. (2015). The influence of the operational environment on efficiency of international airports. Journal of Advanced Transportation, 49(4), 511-522.

Marques, R.C. \& Barros, C.P. (2011). Performance of European airports: regulation, ownership and managerial efficiency. Applied Economics Letters, 18(1), 29-37.

Martini, G.; Manello, A. \& Scotti, D. (2013). The influence of fleet mix, ownership and LCCs on airports'technical/environmental efficiency. Transportation Research Part E, 50(1), 37-52.

Martín, J. \& Roman, C. (2001). An application of DEA to measure the efficiency of Spanish airports prior to privatization. Journal of Air Transport Management, 7, 149-157.

Mendiola, A.; Arévalo, G.; Maratuech, P.; Pérez, J. \& Valencia, J.C. (2011). Concesión del aeropuerto Jorge Chávez: evaluación del valor generado. Lima: ESAN Ediciones.

Oum, T. H.; Yan, J. \& Yu, C. (2008). Ownership forms matter for airport efficiency: a stochastic frontier investigation of worldwide airports. Journal of Urban Economics, 64(2), 422-435.

Oum, T.H.; Adler, N. \& Yu, C. (2006). Privatization, corporatization, ownership forms and their effects on the performance of the world's major airports. Journal of Air Transport Management, 12(3), 109-121.

Oum, T.; Yu, C. \& Fu, X. (2003). A comparative analysis of productivity performance of the world's major airports: summary report of the ATRS global airport benchmarking research report 2002. Journal of Air Transport Management, 9, 285-297.

Oum, T.; Zhang, A. \& Zhang, Y. (2004). Alternative forms of economic regulation and their efficiency implications for airports. Journal of Transport Economics and Policy, 38, 217-246.

Pacheco, R. \& Fernandes, E. (2003). Managerial efficiency of Brazilian airports. Transportation Research Part A, 37, 667-680. 
Parker, D. (1999). The performance of BAA before and after privatization. Journal of Transport Economics and Policy, $33,133-145$.

Pels, E.; Nijkamp, P. \& Rietveld, P. (2001). Relative efficiency of European airports. Transport Policy, 8, 183-192.

Pels, E.; Nijkamp, P. \& Rietveld, P. (2003). Inefficiencies and scale economies of European airport operations.

Transportation Research Part E, 39, 341-361.

Perelman, S. \& Serebrisky, T. (2012). Measuring the technical efficiency of airports in Latin America. Utilities Policy, 22, 1-7.

Perico, A. E.; Santana, N. B. \& Rebelatto, D. A. N. (2017). Eficiência dos aeroportos internacionais brasileiros: uma análise envoltória de dados com bootstrap. Gest. Prod., 24(2), 370-381.

Perico, A. E.; Santana, N. B. \& Capelato, E. (2015). Eficiência Financeira dos Aeroportos Brasileiros: uma análise envoltória de dados. GEPROS - Gestão da Produção, Operações e Sistemas, 10(3), 83-96.

Rhodes, E. (1978). Data envelopment analysis and approaches for measuring the efficiency of decision-making units with an application to program follow-through in U.S. education (Ph.D. dissertation). Carnegie-Mellon University,

Pennsylvania.

Rico Galeana, O. (2008). The privatization of Mexican airports. Journal of Air Transport Management, 14(6), 320-323.

Sarkis, J. \& Talluri, S. (2004). Performance based clustering for benchmarking of US airports. Transportation Research Part A, 38, 329-346.

Sarkis, J. (2000). An analysis of the operational efficiency of major airports in the United States. Journal of Operations Management, 18, 335-51.

Serebrisky, T. (2012). Airport Economics in Latin America and the Caribbean. Washington D.C.: The World Bank.

Thompson, R.; Langemeier, L.; Lee, C.; Lee, E. \& Thrall, R. (1990). The Role of Multiplier Bounds in Efficiency Analysis with Application to Kansas Farming. Econometrics, 46(1), 93-108.

Thompson, R.; Singleton, E.; Thrall, R. \& Smith, B. (1986). Comparative Site Evaluations for Locating High Energy Physics Lab in Texas. Interfaces, 16(6), 35-49.

Tsui, W.; Balli, H.; Gilbey, A. \& Gow, H. (2014). Operational efficiency of Asia-Pacific airports. Journal of Air Transport Management, 40, 16-24.

Ülkü, T. (2015). A comparative efficiency analysis of Spanish and Turkish airports. Journal of Air Transport Management, 46, 56-68

Wanke, P.; Barros, C. \& Nwaogbe, O. (2016). Assessing productive efficiency in Nigerian airports using Fuzzy-DEA. Transport Policy, 49, 9-19.

Wanke, P.F. (2013). Physical infrastructure and flight consolidation efficiency drivers in Brazilian airports: a two-stage network-DEA approach. Journal Air Transport Management, 31, 1-5.

Yang, H.H. (2010a). Measuring the efficiency of Asia-Pacific international airports-Parametric and non-parametric evidence. Computers \& Industrial Engineering, 59(4), 697-702.

Yang, H.H. (2010b). Efficiency and productivity evidence from international airports in the Asia-Pacific region. Journal of the Chinese Institute of Industrial Engineers, 27(2), 157-168.

Yoshida, Y. \& Fujimoto, H. (2004). Japanese-airport benchmarking with the DEA and endogenous-weight TFP methods: testing the criticism of overinvestment in Japanese regional airports. Transportation Research Part E, 40, 533-546.

Editor: Fábio Duarte

Recibido: Nov. 22, 2018

Aprobado: Jun. 4, 2019 